\title{
Cost Effectiveness of Screening for Hepatitis C Virus in Iraq in the Era of Simplified Testing and Treatment
}

\author{
Bassem Asker ${ }^{1} \cdot$ Raghad Jawad $^{1} \cdot$ Rabah Asreah $^{2} \cdot$ Haydar Jamal $^{3} \cdot$ Ahmed Jassem $^{4} \cdot$ Muslim Abdelkareem Inaya $^{4}$. \\ Hiwa Abou Baker ${ }^{3}$. Sam Kozma ${ }^{5}$. Eid Mansour ${ }^{5}$. Bryony McNamara ${ }^{6} \cdot$ Ryan Miller $^{6}$. Oliver Darlington ${ }^{6}$. \\ Phil McEwan $^{6} \cdot$ Daniel M. Sugrue $^{6} \cdot$ Haidar Jarallah $^{7}$
}

Accepted: 28 June 2021 / Published online: 16 August 2021

(c) The Author(s) 2021

\begin{abstract}
Background and Objective Recent advances in hepatitis $\mathrm{C}$ virus (HCV) diagnostic testing methods allow for a one-stop simplified 'test and cure' approach. The cost effectiveness of incorporating this simplified approach into HCV screening in Iraq remains uncertain. This study aimed to compare the cost effectiveness of different HCV testing and diagnostic approaches, and screening strategies in Iraq from a health service perspective.

Methods A cost-effectiveness analysis was undertaken using a hybrid model comprising a screening decision tree linked to a lifetime Markov model to estimate outcomes in $\mathrm{HCV}$-infected people. Cost and utility estimates were sourced from the published literature and expert guidance provided by clinicians and policy makers in Iraq. Cost estimates were reported in 2019 USD or 2019 Iraqi Dinar and both costs and benefits were discounted at 3.5\% annually.

Results Strategies using a simplified approach were found to be cost saving in addition to improving patient outcomes when compared with a standard testing and diagnostic approach. When considering risk-based screening, a simplified approach was associated with a total cost saving of Iraqi Dinar 4375 billion (USD 3.7 billion) and per patient life-year and qualityadjusted life-year gains of 0.30 and 0.55 , compared with a standard approach. Benefits and cost savings were driven by a $32.2 \%$ and $23.6 \%$ reduction in the incidence of cirrhosis and hepatocellular carcinoma, respectively. Estimated benefits and cost savings increased under total population screening. All screening and testing and diagnostic approaches were cost effective compared with a no screening scenario.

Conclusions Improvements in the detection of HCV combined with a simplified one-stop testing and diagnostic approach represents an opportunity to reduce the burden of HCV in Iraq and may play a significant role in meeting World Health Organisation HCV elimination targets.
\end{abstract}

\section{Introduction}

Chronic hepatitis $\mathrm{C}$ virus (HCV) infection is a major public health burden that is associated with an increased risk of liver-related morbidity and mortality [1]. The World Health

Daniel M. Sugrue

daniel.sugrue@heor.co.uk

GIT Center, Baghdad, Iraq

2 Baghdad Teaching Hospital, Baghdad, Iraq

3 Sulaimania Hospital, Sulaimania, Iraq

Najaf Hospital, Najaf, Iraq

5 Gilead Sciences, Dubai, United Arab Emirates

6 Health Economics and Outcomes Research Ltd, Cardiff, UK

7 Basra Hospital, Basra, Iraq
Organisation (WHO) has estimated that 71 million people are infected with $\mathrm{HCV}$ of whom approximately $90 \%$ reside in low-income to middle-income countries [2]. In the developing world, rates of infection are decreasing; however, the burden associated with HCV-related liver disease is expected to increase because of the latent nature of early infection and the large number of people who remain either unaware of their HCV infection status or untreated [3-5].

In 2016, the WHO set a goal to eliminate HCV as a major public health threat by 2030 [6]. However, a number of factors act as a significant barrier to achieving elimination; most notably, the identification of patients and their subsequent engagement and maintenance with their treatment plan $[7,8]$. To address this issue, management frameworks have emerged simplifying the HCV care continuum and promoting easier diagnosis and engagement with medical services $[9,10]$. Increasing the number of people aware 


\section{Key Points for decision makers}

The incorporation of a simplified one-stop 'test and cure' approach into a screening programme is cost effective compared with a standard multi-visit approach as more patients are effectively engaged in care as a result of fewer patients being lost to follow-up between visits

The greater the short-term investment in the diagnosis and treatment of hepatitis $\mathrm{C}$ virus, the greater the longterm savings that can be achieved as a result of costs saved from reduced end-stage liver disease complications

A simplified one-stop testing and diagnostic approach represents an opportunity to broaden screening strategies to people outside of traditional healthcare structures and can play a significant role in meeting World Health Organisation hepatitis $\mathrm{C}$ virus elimination targets

of their HCV status and successfully engaging them in care is likely to improve outcomes in patients with $\mathrm{HCV}$ infection and avoid future infections, thus reducing the burden of disease from both a patient and public health perspective [11-13].

Hepatitis $\mathrm{C}$ virus diagnosis relies on an initial serological test to detect circulating antibodies, which indicates exposure to the virus. To diagnose the active infection, this needs to be followed by nucleic acid testing to detect the presence of the virus in the blood. In the standard diagnostic pathway, $\mathrm{HCV}$ antibodies are detected using a serum or plasma enzyme immunoassay with laboratory test results available after 1 week [14]. Following a positive result, $\mathrm{HCV}$ ribonucleic acid (RNA) testing using a polymerase chain reaction technique is required to detect the active infection [14]. This standard diagnostic pathway requires multiple clinic visits including two phlebotomies (one for an antibody test and one for HCV RNA) with up to $30 \%$ of $\mathrm{HCV}$ antibody-positive patients lost to follow-up between these steps, resulting in a lack of linkage to care and treatment $[15,16]$. Furthermore, there is a long turnaround time and high cost associated with these laboratory-based assays [14].

Recent advances in both serological and molecular testing now allow for a more simplified and efficient approach and include new rapid tests for HCV antibodies (in place of existing classical enzyme immunoassay methods) using serum, plasma, fingerstick whole blood or saliva, and new HCV RNA rapid testing, which can be performed with 100 $\mu \mathrm{L}$ of capillary whole blood, with results available within 1 $\mathrm{h}[14,17]$. By providing rapid results, further investigation and treatment can be offered on the same day during a single visit, before the patient is lost to follow-up, at the point of care by healthcare professionals in the community, in general physicians' practices, sexual health clinics or mobile clinics $[17,18]$. The combination of a simplified diagnostic pathway alongside the initiation of treatment on the same day with pan-genotypic direct-acting antivirals (DAAs) that are capable of achieving cure rates in $99 \%$ of patients [13, 19], a test and cure approach [18], is likely to improve the detection and early initiation of HCV treatment even in those considered difficult to treat because of disease severity or chaotic lifestyles, geography (such as people living in rural areas) or low resource healthcare systems $[17,18]$.

To achieve the WHO's aim of eliminating HCV as a public health threat by 2030 , there needs to be an increased uptake of screening and treatment in countries that historically have not prioritised HCV treatment. Iraq is one such country that has historically not been able to prioritise the implementation of a successful programme for the identification and treatment for HCV driven by numerous challenges such as the availability of funds, the security situation within the country, and overcrowding in the areas of migrants and refugees [20]. However, in recent years, policies have been put in place with the aim of addressing viral hepatitis. In 2013, there was an expansion of diagnostic services from public health laboratories in governorates to district laboratories, which has acted to expand access and opportunities for the testing and diagnosis of HCV [20]. In addition, recent priorities of the Ministry of Health are to establish an efficient hepatitis surveillance system, build the capacity of health personnel to manage patients, and provide medicines and diagnostic services at a governorate level [20]. The implementation of these programmes in addition to improvements in diagnostic approaches and treatments could allow for the effective elimination of HCV in Iraq, provided patients can be effectively identified and engaged in care.

This study aimed to develop an economic model to quantify the predicted long-term clinical and health economic outcomes associated with different screening approaches for HCV infection in Iraq. In particular, the study sought to estimate the costs, the additional number of years of life that a person lives as a result of receiving a treatment (life-years [LYs]), and the length of life adjusted to reflect the quality of life (quality-adjusted life-years [QALYs]) associated with screening, treatment and disease progression. It is envisaged that the results will help inform policy makers and healthcare payers of the expected additional value of a simplified approach to $\mathrm{HCV}$ testing and cure. 


\section{Methods}

\subsection{Study Design}

A cost-effectiveness analysis was undertaken using a hybrid model comprising a screening decision tree linked to a lifetime Markov cohort HCV model built in Microsoft Excel, with inputs informed by the literature and expert opinion. The screening decision tree was designed to estimate the number of HCV-positive people identified at the population level and the cost of achieving this. The HCV Markov model was then used to estimate the direct lifetime healthcare costs, LYs and QALYs in treated and untreated populations. The average age of the cohort at initiation was 43 years, which was informed from the literature and represented the reported average age of assessment across natural history studies of individuals with chronic HCV infection [21]. The model flow diagram is presented in Fig. 1.

\subsection{Screening Decision Tree}

The overall HCV viraemic prevalence in Iraq was estimated at $0.2 \%$ (78,620 persons) and informed from the literature [22]. The proportion of the population that tests positive for HCV antibodies (2.15\%) and HCV RNA (79\%) in highrisk groups was informed by expert opinion as described in Sect. 2.4 (Fig. S1 and Table S2 of the Electronic Supplementary Material [ESM]).

Two screening strategies were assessed: risk-based screening and total population screening. In the risk-based screening approach (indicative of current practice), screening is targeted to specific high-risk populations (pre-marital screening, pre-surgical screening, history of blood transfusion) identified via their routine interaction with the healthcare system. This was estimated to involve screening $3,494,120$ people in year 1 , reflecting $16.4 \%$ of the adult population in Iraq [23], based on the annual number of marriages [24], blood donations [25] and surgical procedures [26] in the adult population of Iraq (Table S1 of the ESM). In total population screening (a hypothetical HCV screening programme), the entire adult population of Iraq was screened in year 1, consisting of $21,266,710$ people [23]. Both screening strategies were assumed to take place within the first year of the modelled scenario and repeated screening was not considered for either strategy.

Each screening strategy was evaluated utilising both standard and simplified testing and diagnostic approaches. In the standard approach, an enzyme immunoassay HCV antibody test and an HCV RNA test (via polymerase chain reaction) are administered during two separate visits, with an assumption of $30 \%$ of patients being lost to follow-up between hospital visits $[15,16]$. In the simplified approach, a rapid HCV antibody test and mobile HCV RNA rapid test are administered during the same visit, with no loss to follow-up assumed to occur. A flow diagram of the two testing and diagnostic approaches considered as part of each screening strategy is provided in Fig. 2. A hypothetical scenario in which there is no screening strategy employed and patients with $\mathrm{HCV}$ are not identified, or treated, was used to estimate the expected cost and health outcomes associated with the progression of the HCV prevalent population. This study therefore considered five scenarios: (1) no screening, (2) risk-based screening with the standard approach to testing and diagnosis (indicative of the current approach used in Iraq), (3) risk-based screening with the simplified approach to testing and diagnosis, (4) total population screening with the standard approach, and (5) total population screening with the simplified approach. A summary of the number of patients who are tested and treated under the different scenarios is presented in Table $\mathrm{S} 2$ of the ESM.

\subsection{Markov Model}

The Markov model was constructed using an established framework for modelling the cost effectiveness of $\mathrm{HCV}$ treatments (Townsend et al. [27] and McEwan et al. [28]) adapted to the Iraq setting. The model has a lifetime horizon (up to 60 years) with a 1-year cycle length and considers only direct healthcare costs to the Iraq health service.

Following a confirmed diagnosis via the screening decision tree, patients entering the model are distributed across METAVIR fibrosis stages F0 (17\%), F1 (35\%), F2 (22\%), F3 (14\%) and F4 (12\%), with the patient distribution informed by a systematic review and meta-analysis of published HCV progression rates [21]. Hepatitis $C$ virus progression is modelled by transitions between health states defined by METAVIR fibrosis stages, complications of end-stage liver disease (ESLD) and all-cause death. State-specific transition probabilities are reported in Table 1 together with annual probabilities of ESLD complications. Costs associated with HCV monitoring and management and patient health-related quality of life are determined by the health state membership.

The model predicts total lifetime costs, LYs, QALYs, the number of predicted liver-related complications and deaths for each screening and testing strategy. Health economic output is also produced including an incremental cost-effectiveness ratio to enable summary comparisons of different strategies where all screening and testing and diagnostic approaches were compared to a no screening scenario. Onward transmission, reinfection and incidence of new HCV infections were not considered. 
Fig. 1 Model flow diagram

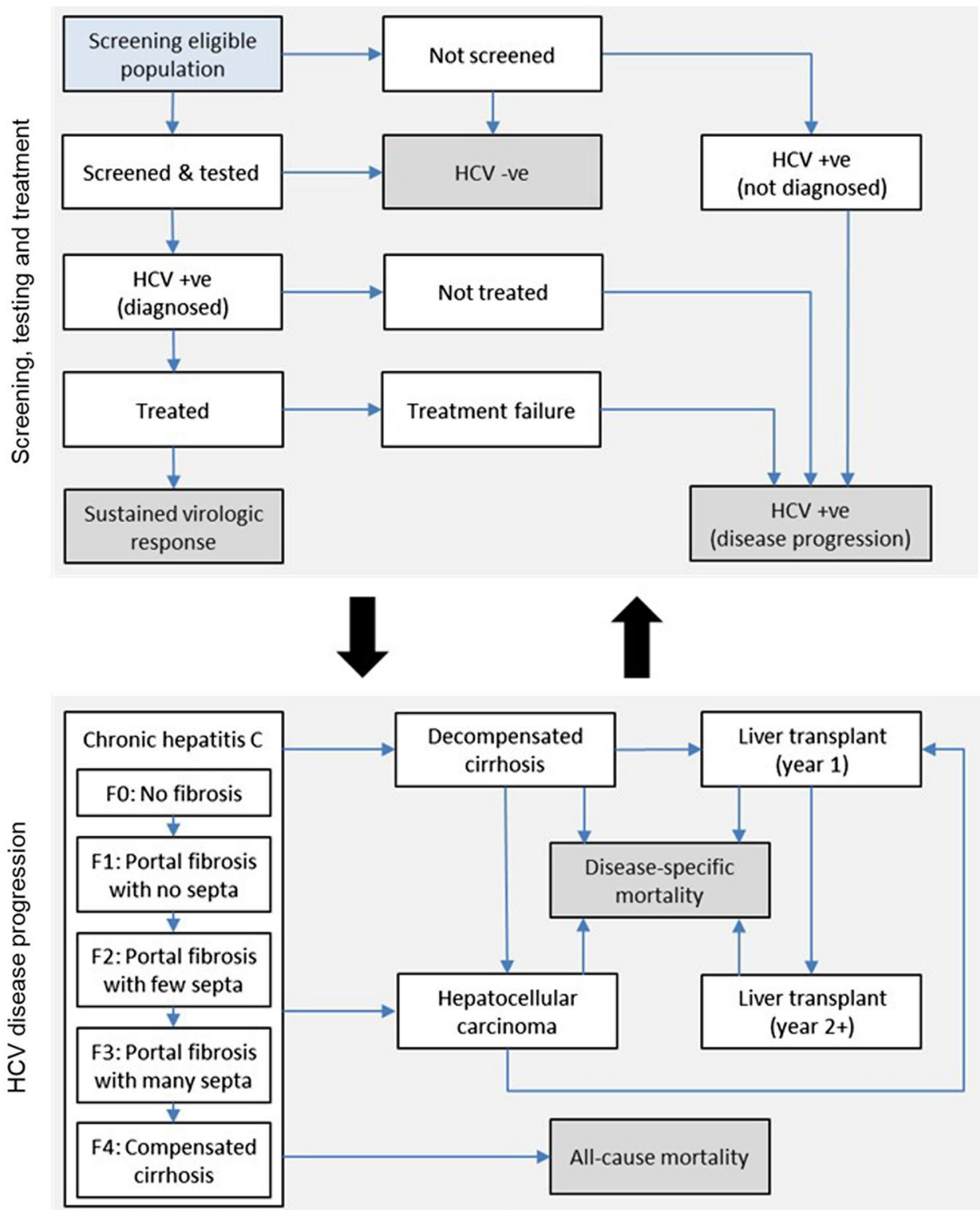

\subsection{Expert Opinion}

Because of limited published data relating to HCV screening and diagnostic strategies and associated costs and resource use in Iraq, a questionnaire was developed to elicit expert opinion from key clinical and policy maker stakeholders. The stakeholder engagement exercise served both to elicit expert opinion on Iraq-specific data that were unavailable in the existing published literature and validate whether initial modelling assumptions were reflective of the Iraqi perspective. Expert opinion was sought to provide model input data for parameters relating to screening, testing, treatment and costs for disease management. Expert opinion was gathered from nine specialist clinicians responsible for the treatment and management of patients with HCV within five Iraqi hospitals and two policy makers responsible for national HCV treatment policy development and treatment procurement in Iraq. Each stakeholder attended a minimum of two faceto-face meetings. The purpose of the first meeting was to demonstrate the questionnaire, explain what was required and answer any questions. During the second face-to-face meeting, the interviewer either guided the stakeholder though the completion of the questionnaire or discussed the completed questionnaire (for those who had completed it ahead of the meeting). Data were reviewed after the first stakeholder completed the exercise to evaluate whether any modifications to the questionnaire were required. Any answer provided by any stakeholder that seemed implausible was queried. Questionnaire results were aggregated, and the final model inputs were an average of the answers provided by all stakeholders for each question. A copy of the questionnaire is provided in the ESM. 

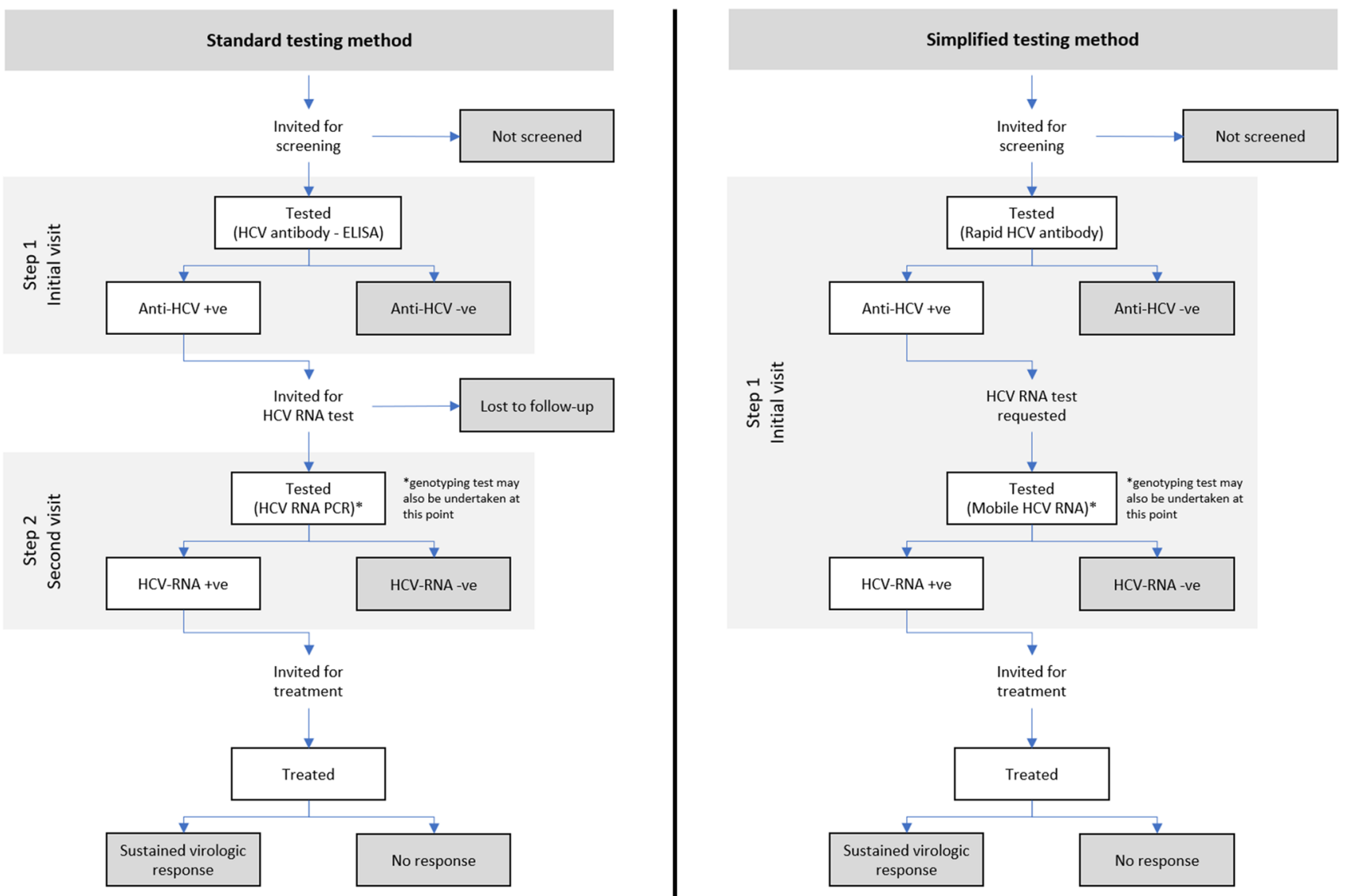

Fig. 2 Flow diagram of the two testing methods considered as part of the screening programme

\subsection{HCV Treatment}

On diagnosis of HCV, patients are allocated treatment according to the specific scenario being considered. It was assumed that all patients identified were treated within the first year as a hypothetical to enable a direct comparison between different screening and testing and diagnostic approaches. Treatment initiation was assumed to be distributed proportionally across METAVIR fibrosis stages. Under base-case assumptions, a pan-genotypic pan-fibrotic DAA with a treatment cure rate of $99 \%$ was assumed under all scenarios [29]. Because of the relatively short treatment period and highly favourable tolerability profiles associated with contemporary HCV therapies, retreatment, treatment-related adverse events, utility decrements and discontinuation were not considered in this analysis.

\subsection{Cost and Health Utility Values}

Analysis was conducted from the Iraq health service perspective considering direct costs only. In the main analysis, costs related to screening and treatment were applied in year 1 as an event cost and treated as an event cost (Table S4 of the ESM). The cost of treatment was based on the price of DAAs currently used in Iraq. The costs of HCV testing and the costs of a course of $\mathrm{HCV}$ treatment were informed by expert opinion or information available from the Iraqi Ministry of Health (Table S4 of the ESM).

Within the HCV Markov model, costs associated with HCV monitoring and management and patient health-related quality of life were applied to patients annually based on their health state (Table 1).The majority of health state costs were informed by expert opinion who were asked to estimate the cost of disease management including administration, testing and monitoring costs, inpatient and outpatient visits, and relevant medication and treatment costs related to the treatment or management of HCV-related conditions. The cost of liver-related death was sourced from a published systematic review [27]. Health state costs of HCV identified by expert opinion were reported in 2019 USD or 2019 Iraqi Dinar (IQD) and were converted appropriately to IQD [30] or USD [30]. The cost of liver-related death informed by the systematic review [27] was reported in 2008 USD, which was inflated to 2018 values [31] and converted to IQD [30].

Health state utility values were taken from the global literature in the absence of Iraqi-specific data (Table 1). 
Table 1 Health state transition rates utility values and costs

\begin{tabular}{|c|c|c|c|c|}
\hline Health state & Input & & Distribution for PSA & Source/comments \\
\hline \multicolumn{5}{|c|}{ Annual transition probability (SE) } \\
\hline $\mathrm{F} 0$ to $\mathrm{F} 1$ & $0.117(0.026)$ & & Beta & Thein et al. [21] \\
\hline $\mathrm{F} 1$ to $\mathrm{F} 2$ & $0.085(0.021)$ & & Beta & Thein et al. [21] \\
\hline $\mathrm{F} 2$ to $\mathrm{F} 3$ & $0.120(0.024)$ & & Beta & Thein et al. [21] \\
\hline $\mathrm{F} 3$ to $\mathrm{F} 4$ & $0.116(0.025)$ & & Beta & Thein et al. [21] \\
\hline $\mathrm{F} 3$ to $\mathrm{DC}$ & $0.000(0.000)$ & & Beta & Assumed \\
\hline F3 to $\mathrm{HCC}$ & $0.002(0.013)$ & & Beta & McEwan et al. [28] \\
\hline F4 to DC & $0.039(0.0039)$ & & Beta & McEwan et al. [13] \\
\hline $\mathrm{F} 4$ to $\mathrm{HCC}$ & $0.014(0.0014)$ & & Beta & McEwan et al. [13] \\
\hline F4 to $\mathrm{LT}$ & $0.000(0.000)$ & & Beta & McEwan et al. [13] \\
\hline $\mathrm{DC}$ to $\mathrm{HCC}$ & $0.014(0.0014)$ & & Beta & McEwan et al. [13] \\
\hline DC to LT & $0.030(0.003)$ & & Beta & McEwan et al. [13] \\
\hline DC (year 1) to death & $0.130(0.013)$ & & Beta & McEwan et al. [13] \\
\hline DC (year 2+) to death & $0.130(0.013)$ & & Beta & Assumed $^{\mathrm{a}}$ \\
\hline $\mathrm{HCC}$ to $\mathrm{LT}$ & $0.040(0.004)$ & & Beta & McEwan et al. [13] \\
\hline HCC (year 1) to death & $0.430(0.043)$ & & Beta & McEwan et al. [13] \\
\hline $\mathrm{HCC}$ (year 2+) to death & $0.430(0.043)$ & & Beta & Assumed $^{\mathrm{a}}$ \\
\hline LT (year 1) to death & $0.210(0.021)$ & & Beta & McEwan et al. [13] \\
\hline LT (year 2+) to death & $0.057(0.0057)$ & & Beta & McEwan et al. [13] \\
\hline F4 post-SVR to DC & $0.001(0.0005)$ & & Beta & McEwan et al. [13] \\
\hline F4 post-SVR to HCC & $0.008(0.0031)$ & & Beta & McEwan et al. [13] \\
\hline \multicolumn{5}{|l|}{ Health state utility } \\
\hline $\mathrm{F} 0$ to $\mathrm{F} 1$ & $0.77(0.016)$ & & Beta & Martin et al. [44] \\
\hline $\mathrm{F} 2$ to $\mathrm{F} 3$ & $0.66(0.03)$ & & Beta & \\
\hline $\mathrm{F} 4$ & $0.55(0.053)$ & & Beta & \\
\hline SVR F0 to F1 & $0.82(0.043)$ & & Beta & \\
\hline SVR F2 to F3 & $0.72(0.05)$ & & Beta & \\
\hline SVR F4 & $0.72(0.05)$ & & Beta & \\
\hline DC & $0.45(0.03)$ & & Beta & \\
\hline $\mathrm{HCC}$ & $0.45(0.03)$ & & Beta & \\
\hline LT (year 1) & $0.45(0.03)$ & & Beta & \\
\hline LT (year 2+) & $0.67(0.067)$ & & Beta & \\
\hline Health state costs & IQD (SE) & USD (SE) & & Source \\
\hline Chronic cirrhosis F0 to F3 & $10,000,000(2,000,000)$ & $8388(1678)$ & Gamma & Expert opinion $^{\mathrm{d}}$ \\
\hline Compensated cirrhosis (F4) & $30,150,000(6,030,000)$ & $25,290(5058)$ & Gamma & Expert opinion $^{\mathrm{d}}$ \\
\hline Decompensated cirrhosis ${ }^{\mathrm{e}}$ & $50,000,000(10,000,000)$ & 41,941 (8388) & Gamma & Expert opinion $^{\mathrm{d}}$ \\
\hline Liver transplant (year 1) & $71,529,600(14,305,920)$ & $60,000(12,000)$ & Gamma & GIT committee $e^{c, g}$ \\
\hline Liver transplant (year 2+) & $1,194,544(238,908)$ & $1002(200)$ & Gamma & GIT committee $e^{c, g}$ \\
\hline Hepatocellular carcinoma ${ }^{\mathrm{f}}$ & $60,000,000(12,000,000)$ & $50,329(10,066)$ & Gamma & Expert opinion $^{\mathrm{d}}$ \\
\hline Liver-related death & $10,567,189(2,113,437)$ & $8841(1768)$ & Gamma & Townsend et al. [27] ${ }^{\mathrm{b}}$ \\
\hline SVR & $300,000(60,000)$ & $252(50.33)$ & Gamma & Expert opinion $^{\mathrm{d}}$ \\
\hline
\end{tabular}

$D C$ decompensated cirrhosis, $F O$ fibrosis stage $0, F 1$ fibrosis stage $1, F 2$ fibrosis stage $2, F 3$ fibrosis stage $3, F 4$ fibrosis stage 4 , GIT gastroenterology and hepatology teaching hospital, $H C C$ hepatocellular carcinoma, $H C V$ hepatitis C virus, IQD Iraqi Dinar, $L T$ liver transplant, $P S A$ probabilistic sensitivity analysis, $S E, S V R$ sustained virologic response, USD US Dollar

${ }^{a}$ Assumed equivalent transition for initial and subsequent years

${ }^{\mathrm{b}}$ A study that performed a systematic review of studies published from 2000 to 2011 to inform key parameters in a cost-effectiveness model of HCV. 2008 USD inflated to 2018 USD using an inflation factor of 1.331 [31], converted to IQD with a conversion of 1,195.22 [30]

${ }^{c} 2019$ USD converted to 2019 IQD with a conversion of 1192.16 (XE Corporation. XE currency converter-live rates: 1 USD to IQD. [Accessed 18/10/2019])

d2019 IQD converted to 2019 USD with a conversion of 0.000838812 (XE Corporation. XE currency converter-live rates: 1 USD to IQD. [Accessed 18/10/2019])

${ }^{\mathrm{e}}$ GIT centre noted DC will go to transplant year 1 India or Turkey

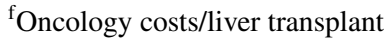

${ }^{\mathrm{g}}$ Committee team at the GIT centre Baghdad 
Age-dependent utility was incorporated within the model to account for decrements in utility as age increases over time. Because of the unavailability of EQ-5D index values based in Iraq, values from the UK were used as a proxy. Costs and benefits are discounted at $3.5 \%$ per annum.

\subsection{Treatment Uptake Analysis}

A treatment uptake analysis was undertaken to explore the impact of alternative capacity assumptions. Assuming all people with HCV are identified, a 10-year time horizon was used to compare the outcomes of patients using a capacity estimate of treating 2000 patients per year, estimated to be the current annual treatment capacity in Iraq informed by expert opinion, against meeting the WHO target of treating $80 \%$ of those HCV-infected by 2030 (6295 patients per year). A hypothetical scenario of treating $100 \%$ of those HCV infected by 2030 was also undertaken ( 7869 patients per year). Patients with decompensated cirrhosis and who had undergone a liver transplant in subsequent years were still considered eligible for treatment with an assumed treatment efficacy of $99 \%$. The cost of treatment reduced in subsequent years to reflect expected cost reductions, informed by expert opinion.

\subsection{Sensitivity Analyses}

Univariable sensitivity analyses were undertaken to assess the most influential factors in estimated incremental costs of total population screening using a simplified approach compared with risk-based screening using a standard approach. A probabilistic sensitivity analysis was conducted across 1000 runs for each screening approach, where inputs are sampled randomly from uncertainty distributions around the mean. Standard errors from data sources were used where possible, otherwise for inputs informed from expert opinion or related to prevalence, a standard error of $20 \%$ of the mean value was used in probabilistic sensitivity analysis sampling.

\subsection{Model Validation}

In line with good practice recommendations, internal validation included an independent review of the model implementation including all input parameters, calculations and codes to ensure accurate implementation [32]. Engagement with clinical experts in Iraq provided us with input in respect of the face validity of the structure, evidence, problem formulation and results of the model. The model was externally validated against a previously published and validated $\mathrm{HCV}$ model (MONARCH [13]) and against a cost-effectiveness study of a birth cohort (1945-65) screening for HCV in the USA. Further detail on the validation of the developed model is available in the ESM.

\section{Results}

\subsection{Health Economic Outcomes of Different HCV Screening Strategies}

The total discounted costs associated with no screening, risk-based screening with either a standard or simplified $\mathrm{HCV}$ testing and diagnostic approach, and total population screening with either a standard or simplified HCV testing and diagnostic approach over a lifetime (approximately 60 years) horizon are presented in Fig. 3 and Table 2. The total cost associated with a no screening scenario was estimated at IQD 24,526 billion [Bn] (USD 20.3 Bn). These costs were largely driven by the management of the disease and ESLD complications, as this scenario assumes curative treatment of HCV does not occur (Table 3). Compared with a no screening scenario, risk-based screening using a standard $\mathrm{HCV}$ testing approach was associated with a cost saving of IQD $9760 \mathrm{Bn}$ (USD 8.2 Bn) with overall costs of IQD 14,496 Bn (USD 12.2 Bn). Screening the total population using a standard HCV testing approach was associated with overall costs of IQD 10,321 Bn (USD 8.7 Bn), a cost saving of IQD 13,935 Bn (USD 11.7 Bn) compared with the no screening scenario (Table 4).

The reduction in costs associated with risk-based screening were also associated with an increase in LYs and QALYs. Using the standard HCV testing approach, discounted per-HCV-infected person LYs increased from 17.48 in the no screening scenario to 18.19 in the riskbased screening scenario, a LY gain of 0.70 years (Fig. S2 of the ESM). Similarly, discounted per-HCV-infected person QALYs increased from 10.46 to 11.74 , a QALY gain of 1.28. Total population-based screening using a standard $\mathrm{HCV}$ testing approach was also associated with an increase in LYs and QALYs compared with no screening. Discounted per-HCV-infected person LYs increased to 18.61 years in the total population-based screening scenario, a LY gain of 1.13 years compared with a no screening scenario. Similarly, discounted per-HCV-infected person QALYs increased to 12.52, a QALY gain of 2.06 .

The introduction of a simplified approach to HCV testing and cure was associated with a reduction in costs and an increase in LYs and QALYs when compared with the standard approach. In the risk-based screening scenario, the simplified HCV testing approach resulted in a discounted cost saving of IQD $4375 \mathrm{Bn}$ (USD 3.7 Bn) and discounted per-HCV-infected person LY and QALY gains of 0.30 and 0.55 , respectively, compared with the standard HCV testing approach. Similarly, the total population screening scenario saw a discounted cost saving of IQD 7177 Bn (USD 6.0 Bn) and discounted HCV-infected person LY and QALY gains of 0.48 and 0.88 , respectively. 


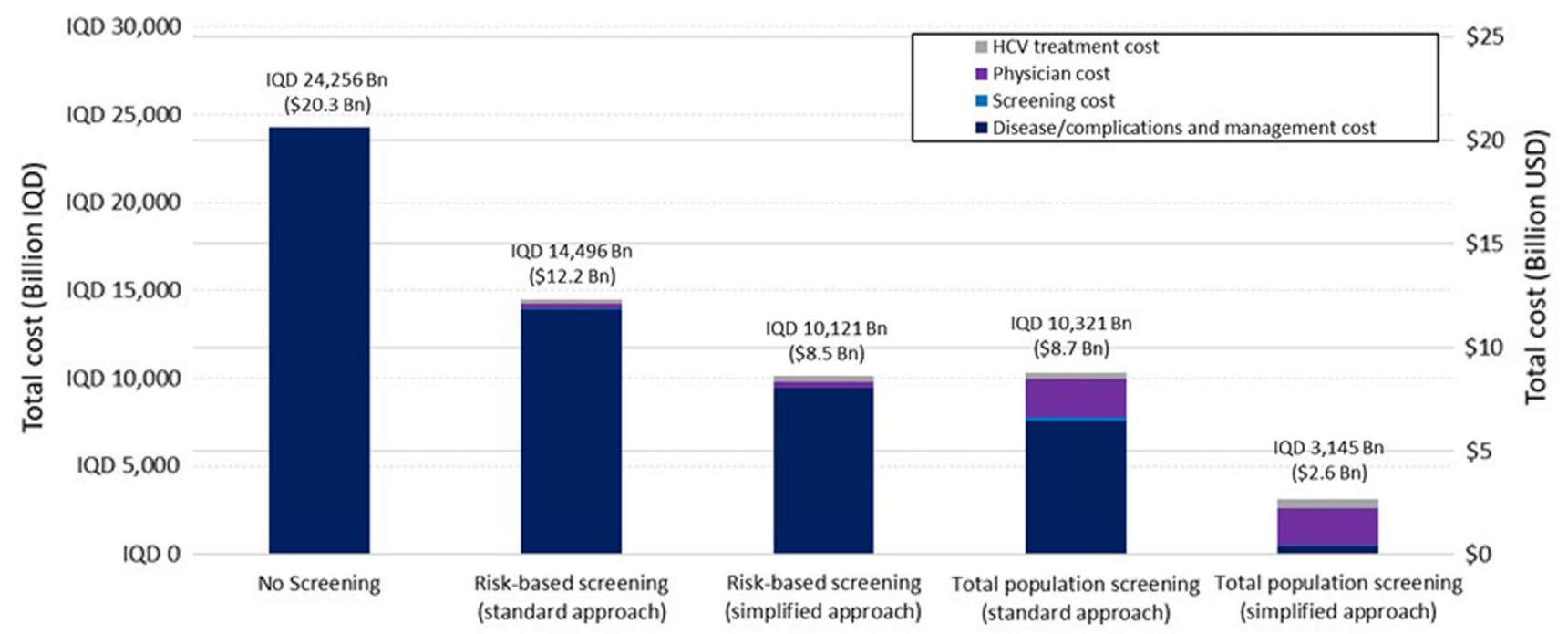

Fig. 3 Total direct healthcare costs over a lifetime (approximately 60 years) horizon (categorised into physicians' fees, and cost of screening, hepatitis $\mathrm{C}$ virus [HCV] treatment and management of the disease and end-stage liver disease [ESLD] complications) for five sce- narios: no screening, risk-based screening with a either a standard or simplified approach, or total population level screening with a either a standard or simplified approach
Table 2 Total direct healthcare costs over a lifetime (approximately 60 years) horizon (partitioned into costs associated with screening, physicians, HCV treatment and disease/complications and manage- ment) for five scenarios: no screening, risk-based screening with a either a standard approach or simplified approach, or total population level screening with a either a standard or simplified approach

\begin{tabular}{|c|c|c|c|c|c|}
\hline & No screening & $\begin{array}{l}\text { Risk-based screen- } \\
\text { ing with a standard } \\
\text { approach }\end{array}$ & $\begin{array}{l}\text { Risk-based screen- } \\
\text { ing with a simplified } \\
\text { approach }\end{array}$ & $\begin{array}{l}\text { Total population screen- } \\
\text { ing with a standard } \\
\text { approach }\end{array}$ & $\begin{array}{l}\text { Total population screening } \\
\text { with a simplified approach }\end{array}$ \\
\hline \multicolumn{6}{|l|}{ IQD (Bn) } \\
\hline Screening cost & - & 36 & 5 & 217 & 27 \\
\hline Physician cost & - & 330 & 326 & 2134 & 2127 \\
\hline $\mathrm{HCV}$ treatment cost & - & 224 & 321 & 361 & 516 \\
\hline $\begin{array}{l}\text { Disease/complica- } \\
\text { tions and manage- } \\
\text { ment cost }\end{array}$ & 24,256 & 13,905 & 9469 & 7609 & 475 \\
\hline Total direct costs & 24,256 & 14,496 & 10,121 & 10,321 & 3145 \\
\hline \multicolumn{6}{|l|}{ USD (Bn) } \\
\hline Screening cost & - & 0.030 & 0.004 & 0.182 & 0.023 \\
\hline Physician cost & - & 0.3 & 0.3 & 1.8 & 1.8 \\
\hline $\mathrm{HCV}$ treatment cost & - & 0.2 & 0.3 & 0.3 & 0.4 \\
\hline $\begin{array}{l}\text { Disease/complica- } \\
\text { tions and manage- } \\
\text { ment cost }\end{array}$ & 20.3 & 11.7 & 7.9 & 6.4 & 0.4 \\
\hline Total direct costs & 20.3 & 12.2 & 8.5 & 8.7 & 2.6 \\
\hline
\end{tabular}

$B n$ billion, $H C V$ hepatitis C virus, IQD Iraqi Dinar, USD United States Dollar

In terms of cost effectiveness, both the risk-based and the total population screening scenarios were dominant compared with a no screening scenario, i.e. the scenarios were less costly and associated with improved health outcomes compared with a no screening scenario, which was largely driven by a reduction in the cost of HCV-related complications. When comparing the two screening scenarios, total population screening was more cost effective than the riskbased screening approach, and a simplified approach was more cost effective than a standard approach (Table 4). 
Table 3 Estimated numbers of ESLD clinical complications in the hepatitis $\mathrm{C}$ virus prevalent population over a lifetime (approximately 60 years) horizon under five scenarios: no screening, risk-based screening with a either a standard or simplified approach, or total population level screening with a either a standard or simplified approach

\begin{tabular}{llllll}
\hline ESLD complication & No screening & $\begin{array}{l}\text { Risk-based screen- } \\
\text { ing with a standard } \\
\text { approach }\end{array}$ & $\begin{array}{l}\text { Risk-based screen- } \\
\text { ing with a simplified } \\
\text { approach }\end{array}$ & $\begin{array}{l}\text { Total population screen- } \\
\text { ing with a standard } \\
\text { approach }\end{array}$ & $\begin{array}{l}\text { Total population screening } \\
\text { with a simplified approach }\end{array}$ \\
\hline CC & 48,327 & 27,503 & 18,578 & 14,836 & 483 \\
DC & 25,863 & 14,832 & 10,105 & 8123 & 520 \\
HCC & 12,170 & 7844 & 5989 & 5212 & 2230 \\
Liver transplant & 5457 & 3203 & 2237 & 1832 & 279 \\
Liver-related death & 34,793 & 20,809 & 14,816 & 12,303 & 2665 \\
\hline
\end{tabular}

$C C$ compensated cirrhosis, $D C$ decompensated cirrhosis, ESLD end-stage liver disease, $H C C$ hepatocellular carcinoma

Table 4 Estimated life-years, QALYs, direct costs and economic outcomes over a lifetime (approximately 60 years) horizon under five scenarios: no screening, risk-based screening with a either a stand- ard or simplified approach, or total population level screening with a either a standard or simplified approach

\begin{tabular}{|c|c|c|c|c|c|}
\hline & No screening & $\begin{array}{l}\text { Risk-based screen- } \\
\text { ing with a standard } \\
\text { approach }\end{array}$ & $\begin{array}{l}\text { Risk-based screen- } \\
\text { ing with a simplified } \\
\text { approach }\end{array}$ & $\begin{array}{l}\text { Total population screening } \\
\text { with a standard approach }\end{array}$ & $\begin{array}{l}\text { Total population screening } \\
\text { with a simplified approach }\end{array}$ \\
\hline Life-years $^{\mathrm{a}}$ & $1,374,629$ & $1,429,874$ & $1,453,551$ & $1,463,479$ & $1,501,558$ \\
\hline QALYs $^{\mathrm{a}}$ & 822,350 & 922,877 & 965,959 & 984,025 & $1,053,314$ \\
\hline \multicolumn{6}{|l|}{ IQD (Bn) } \\
\hline Total direct costs & 24,256 & 14,496 & 10,121 & 10,321 & 3145 \\
\hline ICER & NA & Dominant $^{\mathrm{b}}$ & Dominant $^{\mathrm{b}}$ & Dominant $^{b}$ & Dominant $^{\mathrm{b}}$ \\
\hline \multicolumn{6}{|l|}{ USD (Bn) } \\
\hline Total direct costs & 20.3 & 12.2 & 8.5 & 8.7 & 2.6 \\
\hline ICER & NA & Dominant $^{\mathrm{b}}$ & Dominant $^{\mathrm{b}}$ & Dominant $^{\mathrm{b}}$ & Dominant $^{\mathrm{b}}$ \\
\hline
\end{tabular}

$B n$ billion, $H C V$ hepatitis $\mathrm{C}$ virus, ICER incremental cost-effectiveness ratio, IQD Iraqi Dinar, $Q A L Y$ quality-adjusted life-year, $U S D$ United States Dollar

${ }^{\text {a }}$ Based on the estimated Iraqi HCV prevalent population (approximately 78,620 )

${ }^{\mathrm{b}} \mathrm{A}$ dominant screening scenario is one that is both less costly and results in better health outcomes than no screening (screening 'dominates' no screening)

\subsection{Clinical Outcomes of Different HCV Screening Strategies}

In addition to the economic benefits, the risk-based and total population screening strategies were associated with substantial clinical benefits via a reduction in HCV-related complications compared with no screening (Table 3 ). The most beneficial scenario was the use of total population screening and a simplified HCV testing and diagnostic approach, which resulted in a 99.0\%, 98.0\%, 81.7\%, 94.9\% and $92.3 \%$ reduction in the incidences of compensated cirrhosis, decompensated cirrhosis, hepatocellular carcinoma, liver transplant and liver-related mortality, respectively, when compared with a no screening scenario over a lifetime (approximately 60 years) horizon. This compares with a $43.1 \%, 42.7 \%, 35.5 \%, 41.3 \%$ and $40.2 \%$ reduction in the incidences of compensated cirrhosis, decompensated cirrhosis, hepatocellular carcinoma, liver transplant and liver-related mortality, respectively, under the risk-based screening strategy using a standard HCV testing approach.

\subsection{Treatment Uptake Analysis}

In a treatment uptake analysis over a 10-year time horizon, no treatment uptake was compared against current treatment uptake rates and treatment uptake rates required to meet the WHO target of treating $80 \%$ of those HCV infected by 2030 , or a hypothetical scenario of treating all $\mathrm{HCV}$-infected persons by 2030. Under current capacity assumptions, treatment uptake would result in $25.4 \%$ of the $\mathrm{HCV}$-infected population being treated, far below the WHO target of treating $80 \%$. Compared with current capacity estimates, increasing capacity and treatment uptake to 6295 persons per year to meet the WHO target resulted in an USD 101.2 million increase in drug costs over 10 years (from USD 47.2 million to USD 148.4 million). However, there were USD $2.1 \mathrm{Bn}$ of 


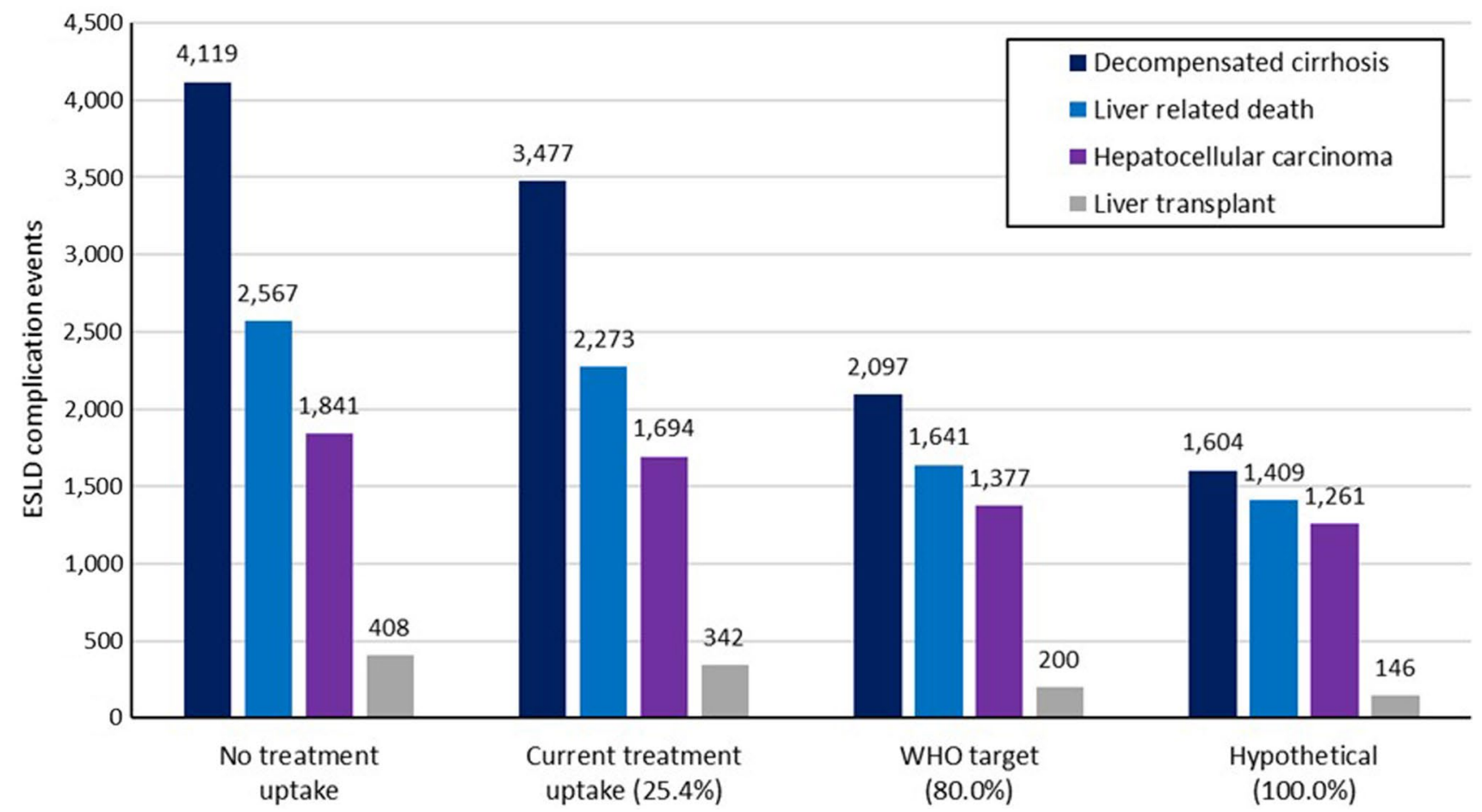

Fig. 4 End-stage liver disease (ESLD) complication events over a 10-year time horizon for four treatment uptake scenarios: no treatment uptake, current treatment uptake, treatment update required to

meet World Health Organisation (WHO) treatment uptake target and a hypothetical scenario treating all hepatitis $\mathrm{C}$ virus (HCV)-infected persons

savings in disease management and complications costs over the 10 years (from USD 6.8 Bn to USD 4.7 Bn). This was because of $39.7 \%$ fewer instances of decompensated cirrhosis (3477 vs 2097), $18.7 \%$ fewer instances of hepatocellular carcinoma (1694 vs 1377), $41.6 \%$ fewer instances of transplant (342 vs 200) and 27.8\% fewer instances of liver-related death (2237 vs 1641), respectively (Fig. 4). Consequently, whilst there were additional costs associated with increased treatment uptake, these are offset by the large reductions in costs associated with ESLD management (Table 5).

\subsection{Sensitivity Analyses}

Univariable sensitivity analyses demonstrated that base-case conclusions remained the same when various factors were adjusted, with the most influential factors in estimated incremental costs being: (1) discounting, (2) HCV prevalence, (3) simplified vs standard testing and diagnostic method, and (4) disease/complications and management costs (Fig. 5). Total population screening using a simplified testing and diagnostic approach was cost saving in all scenarios in comparison with risk-based screening using a standard approach. In the probabilistic sensitivity analysis, risk-based screening strategies were cost effective $98.80 \%+$ of the time, and total population strategies were cost effective in $100 \%$ of the runs at the threshold of 9,000,000 IQD (ESM).

\subsection{Model Validation}

The predicted outcomes of the HCV model align closely with the outcomes from the previously validated MONARCH model, as evidenced by the R2 range from 0.941 to 0.959 . The model also validated well to outcomes of a costeffectiveness study of a birth cohort (1945-65) screening for HCV in the USA. [33] Further detail on the validation of the developed model is available in the ESM.

\section{Discussion}

This study demonstrates that screening the population of Iraq for HCV is cost effective, achieving higher cost savings and QALY gains compared with the current risk-based screening practice. The cost effectiveness was driven by the successful treatment of patients shortly after $\mathrm{HCV}$ diagnosis and the avoidance of downstream HCV-related complications, which are associated with significant reductions in quality of life and significantly increased medical expenditure. Screening strategies using a simplified approach were found to be the most cost effective, driven not only by the reduced costs of the diagnostic tests and the fewer clinical visits, but also because of more patients being effectively engaged in care and fewer lost to follow-up, as is observed 
Table 5 Total direct healthcare costs over a 10 -year time horizon (partitioned into costs associated with screening, physicians, HCV treatment and disease/complications and management) for four scenarios: no treatment uptake, current treatment uptake, treatment uptake aligned to WHO elimination targets and treatment uptake by the entire $\mathrm{HCV}$-infected population

\begin{tabular}{cllll}
\hline & No treatment uptake & $\begin{array}{l}\text { Current treatment } \\
\text { uptake }(25.4 \% \\
\text { treated })\end{array}$ & $\begin{array}{l}\text { WHO target } \\
(80 \% \text { treated })\end{array}$ & $\begin{array}{l}\text { Hypothetical } \\
\text { scenario }(100 \% \\
\text { treated })\end{array}$ \\
\hline $\begin{array}{l}\text { IQD } \\
\text { HCV treatment cost }\end{array}$ & - & $56.2 \mathrm{Bn}$ & $176.9 \mathrm{Bn}$ & $218.6 \mathrm{Bn}$ \\
$\begin{array}{c}\text { Disease/complica- } \\
\text { tions and manage- } \\
\text { ment cost }\end{array}$ & $9309.6 \mathrm{Bn}$ & $8144.5 \mathrm{Bn}$ & $5642.6 \mathrm{Bn}$ & $4742.2 \mathrm{Bn}$ \\
$\begin{array}{c}\text { USD } \\
\begin{array}{c}\text { HCV treatment cost } \\
\text { Disease/complica- } \\
\text { tions and manage- } \\
\text { ment cost }\end{array}\end{array}$ & - & & & \\
\hline
\end{tabular}

$B n$ billion, $H C V$ hepatitis $\mathrm{C}$ virus, $I Q D$ Iraqi Dinar, $M$ million, USD United States Dollar, WHO World Health Organisation
Cost discounting $[0.0 \%, 5.0 \%]$

HCV prevalence [mean $+/-20 \%]$

Disease/complications and management cost [mean $+/-20 \%]$

Simplified testing $[0 \%, 100 \%]^{*}$

Proportion currently screened [mean $+/-20 \%]^{*}$

Treatment efficacy [mean $+/-20 \%]$

Proportion LTF prior to HCV RNA test [mean +/- 20\%]*

Proportion treated [mean $+/-20 \%]$

Physician cost [mean $+/-20 \%]$

Time horizon [ 40 years]

HCV treatment cost [mean $+/-20 \%]$

Screening cost [mean $+/-20 \%]$

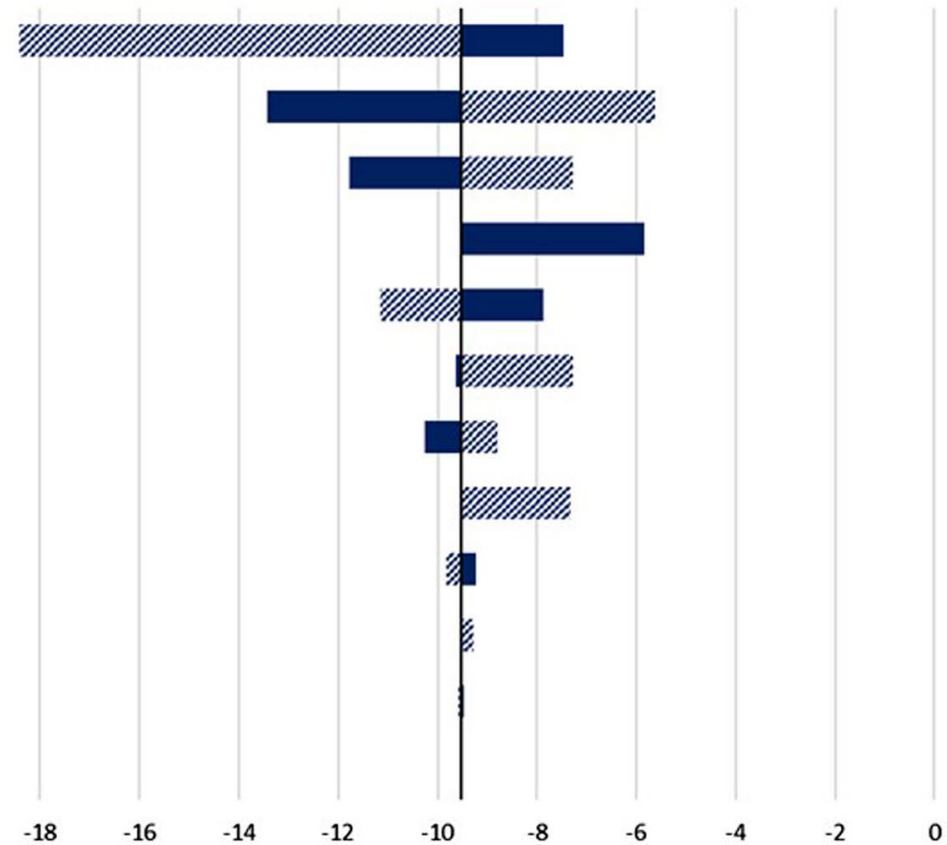

Incremental costs (Billion USD) - total population (simplified) vs. risk-based (standard) screening

* Only applied to the current screening arm

\& Lower bound upper bound

Fig. 5 Deterministic sensitivity analysis

in the standard pathway between antibody and HCV RNA testing. The key findings of this study are highlighted in Box 1 to help inform policy makers and payers of the health economic value of screening for $\mathrm{HCV}$ in Iraq using a simplified approach to $\mathrm{HCV}$ testing and cure.

Because of the global prevalence of $\mathrm{HCV}$, there has been considerable interest in assessing the cost effectiveness of screening programmes for $\mathrm{HCV}$; with analyses performed in several countries [28, 34-38]. However, countries have different needs and face different realities as they confront their HCV epidemics. Consequently, challenges that face one country, such as Iraq, are likely to differ to those challenges that face another country, such as the USA, and consideration should be given to country-specific challenges when comparing the cost effectiveness of screening strategies. Indeed, while access to affordable treatment is key, analysis from the WHO has shown that countries need a strong government support, national plans for preventing, 
Box 1 Summary of key findings

The World Health Organisation targets for 2030 are to achieve a $90 \%$ reduction in new hepatitis C virus (HCV) infections, treatment of $80 \%$ of treatment-eligible people with chronic hepatitis and a $65 \%$ reduction in HCV liver-related deaths

Availability of point-of-care rapid tests means that HCV antibody screening and confirmatory ribonucleic acid can now be achieved in a single day, resulting in immediate initiation of treatment and a one-stop test and cure approach for $\mathrm{HCV}$

Results of this study demonstrate that using current standard HCV diagnostic testing methods, screening the total Iraqi population was more cost effective than no screening or risk-based screening (costs saved from reduced end-stage liver disease-related complications outweighed the additional costs of screening and treatment), and was also associated with increases in life-years and quality-adjusted life-years

The introduction of a simplified approach was associated with a significant reduction in costs and HCV-related complications and an increase in life-years and quality-adjusted life-years when compared with the standard testing and diagnostic approach

In addition to the economic benefits, the screening strategies were associated with substantial clinical benefits including a reduction in HCVrelated complications such as cirrhosis, hepatocellular carcinoma and liver-related mortality

Treatment uptake in line with the World Health Organisation treatment target resulted in a total cost saving of USD 2.1 billion over 10 years in comparison with current treatment capacity estimates. Cost savings were driven by $39.7 \%$ fewer instances of decompensated cirrhosis ( 3477 vs 2097), $18.7 \%$ fewer instances of hepatocellular carcinoma (1694 vs 1377), 41.6\% fewer instances of transplant (342 vs 200) and $27.8 \%$ fewer instances of liver-related death (2237 vs 1641), respectively

HCV screening using a simplified testing and diagnostic approach represents an opportunity to broaden screening strategies to people outside of traditional healthcare structures and dramatically reduce the burden of HCV in Iraq

diagnosing and treating $\mathrm{HCV}$, and adequate financing to roll out and sustain HCV services, which all differ across countries [39].

Despite the additional disease management and treatment cost incurred as a result of identifying many previously undiagnosed patients with $\mathrm{HCV}$, screening and treatment strategies in Iraq remain highly cost effective. Supplementary analysis demonstrates that the identification of further individuals with $\mathrm{HCV}$, by increasing the number of individuals who are screened, results in additional cost savings (Fig. S3 of the ESM) and QALY gains (Fig. S4 of the ESM). However, the implementation of any screening programme comes with important considerations. For example, any upscaling of testing requires simultaneous investment in direct linkage to care and treatment, which would require considerable capital investment as well as an update in clinical practice, improved patient engagement and an expansion in services. However, the potential cost offset by reducing the costs associated with managing $\mathrm{HCV}$ and subsequent ESLD would be considerable and arguably justifies such investments.

Numerous studies have demonstrated that HCV therapy and the achievement of sustained virologic response results in dramatic decreases in hepatic decompensation events, hepatocellular carcinoma and liver-related mortality [3-5]. Increased treatment uptake has also been shown to be a key driver of future infections avoided [40], which brings with it further public health benefits from the avoidance of future infections and subsequent avoidance of long-term complications of $\mathrm{HCV}$ and increased treatment cost offsets [41]. However, if $\mathrm{HCV}$ elimination is to be considered a realistic clinical goal, a key challenge will be the identification and treatment of those additional patients who are not currently seen by healthcare professionals, for example patients unaware of their infection, and those disinclined to engage with health services.
A notable limitation of this study relates to uncertainty around input estimates as there was a general paucity of Iraq-specific data available with which to parameterise the model. While inputs related to disease progression, such as transition rates, are unlikely to differ between countries, epidemiological and cost estimates are likely to differ between settings. To fill some of these evidence gaps, a survey to elicit expert opinion from clinicians and policy makers was undertaken. However, uncertainty remains, particularly with regard to Iraq-specific cost estimates. Epidemiological data such as the distribution of the fibrosis stage and treatment in people with $\mathrm{HCV}$ in Iraq and the prevalence of $\mathrm{HCV}$ in at-risk populations is also limited; current analysis considered the pre-marital population, those undergoing surgery and those with a history of blood transfusion to be at a high risk of HCV infection. However, this is indicative of a current approach only and it is also likely that there are other high-risk populations that have not been considered in these analyses, such as those who are incarcerated, injectable drug users, those with HIV, and those undergoing dialysis or receiving other haematological products. An additional limitation is that this study did not consider onward transmission, reinfection and the incidence of new HCV infections, which would be impacted by improved diagnosis and treatment of $\mathrm{HCV}$; consequently, results are likely to underestimate the public health benefit and cost savings associated with the avoidance of future infections. This was made as a simplifying assumption because of the lack of Iraq-specific data to inform relevant inputs.

While some of our scenarios are not practical to implement under current assumptions (e.g. screening and treatment in year 1), results show that the greater the short-term investment in the diagnosis and treatment of $\mathrm{HCV}$, the greater savings, LYs and quality of life is achieved. Results 
also suggest that a national screening programme in Iraq, and in particular, a screening strategy incorporating a simplified testing and diagnostic approach that supports the goal of a one-stop 'test and cure' for HCV (i.e. diagnostic testing with initiation of treatment for positive patients in the same clinic visit), could significantly reduce the incidence of HCV-related ESLD complications and mortality, reduce costs and offer an important initial step towards the formation of a national health policy aimed at managing $\mathrm{HCV}$ in the Iraqi population. The next step is to determine how this could be best achieved in real-world practice as such an approach to an HCV test and cure screening programme would require significant investment and further discussion on the wider feasibility and affordability is required. However, such an investment is likely to be an efficient costeffective approach and an important contribution to the WHO's global health strategy to eliminate $\mathrm{HCV}$ as a major public health threat by 2030 [42] and real-world experience has shown that with political will and affordable pricing, decentralised HCV programmes are achievable in resourcelimited settings [43]. Further research into the optimisation of risk-based screening strategies in which additional patients beyond current practice are screened and identified is also needed to fully explore the potential benefits of this approach to the HCV test and cure.

\section{Conclusions}

This study suggests the incorporation of a simplified approach into HCV screening in Iraq is cost effective compared with a standard multi-visit approach driven by increases in LYs and QALYs and costs saved from reduced ESLD-related complications that outweigh the additional costs of screening and treatment. A one-stop 'test and cure' approach also provides an opportunity to broaden screening strategies to people outside of traditional healthcare structures allowing more people to be identified and effectively engaged in care, which could lead to a reduction in the burden of $\mathrm{HCV}$ in Iraq.

Supplementary Information The online version contains supplementary material available at https://doi.org/10.1007/s40273-021-01064-z.

Acknowledgements The authors thank Mr. Mohammed Imad and Dr. Abdullah Abdul Rahman Khaild for conducting interviews with participating stakeholders.

\section{Declarations}

Funding Gilead Sciences provided support for this study. The funding agreement ensured the authors' independence in designing the study, interpreting the data and preparing the manuscript for publication.
Conflict of interest Daniel M. Sugrue, Ryan Miller, Oliver Darlington, Bryony McNamara and Phil McEwan are employees of Health Economics and Outcomes Research Ltd. and received funding from Gilead Sciences to undertake the research outlined in this study. Sam Kozma and Eid Mansour are employees of Gilead Sciences. Bassem Asker, Rabah Asreah, Hiwa Abou Baker, Muslim Abdelkareem Inaya, Haydar Jamal, Haidar Jarallah, Ahmed Jassem and Raghad Jawad have no conflicts that are directly relevant to the content of this article.

Ethics approval Not applicable.

Consent to participate Not applicable.

Consent for publication Not applicable.

Availability of data and material All data generated or analysed during this study are included in this published article and its supplementary information files.

Code availability The code associated with this study is proprietary.

Authors' contributions All authors contributed to the design of the research and ensuring the final content was reflective of the Iraq setting. DS, BM and SK led the design and implementation of the research and to the writing of the manuscript. RM contributed to the design and implementation of the research, to the analysis of the data and to the writing of the manuscript. OD and PM provided senior review and technical expertise and contributed to the design of the research and to the writing of the manuscript. EM provided senior review and contributed to the design and implementation of the research. All named authors meet the International Committee of Medical Journal Editors criteria for authorship for this manuscript, take responsibility for the integrity of the work as a whole and have given final approval to the version to be published. As the guarantor and corresponding author, DS takes full responsibility for the work as a whole, and the decision to submit and publish the manuscript.

Open Access This article is licensed under a Creative Commons Attribution-NonCommercial 4.0 International License, which permits any non-commercial use, sharing, adaptation, distribution and reproduction in any medium or format, as long as you give appropriate credit to the original author(s) and the source, provide a link to the Creative Commons licence, and indicate if changes were made. The images or other third party material in this article are included in the article's Creative Commons licence, unless indicated otherwise in a credit line to the material. If material is not included in the article's Creative Commons licence and your intended use is not permitted by statutory regulation or exceeds the permitted use, you will need to obtain permission directly from the copyright holder. To view a copy of this licence, visit http://creativecommons.org/licenses/by-nc/4.0/.

\section{References}

1. Poynard T, Yuen MF, Ratziu V, Lai CL. Viral hepatitis C. Lancet. 2003;362(9401):2095-100.

2. Stanaway JD, Flaxman AD, Naghavi M, Fitzmaurice C, Vos T, Abubakar I, et al. The global burden of viral hepatitis from 1990 to 2013: findings from the Global Burden of Disease Study 2013. Lancet. 2016;388(10049):1081-8.

3. Cheung MCM, Walker AJ, Hudson BE, Verma S, McLauchlan J, Mutimer DJ, et al. Outcomes after successful direct-acting antiviral therapy for patients with chronic hepatitis $\mathrm{C}$ and decompensated cirrhosis. J Hepatol. 2016;65(4):741-7. 
4. Hallager S, Ladelund S, Christensen PB, Kjær M, Thorup Roege B, Grønbæk KE, et al. Liver-related morbidity and mortality in patients with chronic hepatitis $\mathrm{C}$ and cirrhosis with and without sustained virologic response. Clin Epidemiol. 2017;9:501-16.

5. Nahon P, Bourcier V, Layese R, Audureau E, Cagnot C, Marcellin $\mathrm{P}$, et al. Eradication of hepatitis $\mathrm{C}$ virus infection in patients with cirrhosis reduces risk of liver and non-liver complications. Gastroenterology. 2017;152(1):142-56 (e2).

6. World Health Organisation. Combating hepatitis B and C to reach elimination by 2030. Geneva: World Health Organisation; 2016.

7. Johnson S, Aluzaite K, Taar A, Schultz M. Identifying barriers to treatment of $\mathrm{HCV}$ in the primary care setting. Hepatol Int. 2019;13(1):58-65.

8. Skeer MR, Ladin K, Wilkins LE, Landy DM, Stopka TJ. "Hep C's like the common cold": understanding barriers along the HCV care continuum among young people who inject drugs. Drug Alcohol Depend. 2018;1(190):246-54.

9. Kapadia SN, Marks KM. Hepatitis C management simplification from test to cure: a framework for primary care providers. Clin Ther. 2018;40(8):1234-45.

10. Dieterich DT. A Simplified algorithm for the management of hepatitis C infection. Gastroenterol Hepatol. 2019;15(5 Suppl. 3):1-12.

11. Ward T, Gordon J, Jones B, Bennett H, Webster S, Kalsekar A, et al. Value of sustained virologic response in patients with hepatitis $\mathrm{C}$ as a function of time to progression of end-stage liver disease. Clin Drug Investig. 2017;37(1):61-70.

12. Ward T, Gordon J, Bennett H, Webster S, Sugrue D, Jones B, et al. Tackling the burden of the hepatitis $\mathrm{C}$ virus in the UK: characterizing and assessing the clinical and economic consequences. Public Health. 2016;141:42-51.

13. McEwan P, Ward T, Bennett H, Kalsekar A, Webster S, Brenner $\mathrm{M}$, et al. Estimating the clinical and economic benefit associated with incremental improvements in sustained virologic response in chronic hepatitis C. PLoS One. 2015;10(1):e0117334.

14. European Association for the Study of the Liver (EASL). EASL recommendations on treatment of hepatitis C 2018. J Hepatol. 2018;69(2):461-511.

15. Munang M, Smit E, Barnett T, Atherton C, Tahir M, Atabani SF. Outcomes and costs of single-step hepatitis C testing in primary care, Birmingham, United Kingdom. Public Health. 2019;166:40-4.

16. Garcia FG, Dominguez R, Arboledas JCA, Casado M, Sanchez JM, Pérez FT, et al. FRI-229-reflex testing in patients with chronic hepatitis C in Spain improves healthcare outcomes and is cost effective. J Hepatol. 2019;70(1):e495-6.

17. Lamoury FMJ, Bajis S, Hajarizadeh B, Marshall AD, Martinello M, Ivanova E, et al. Evaluation of the Xpert HCV viral load finger-stick point-of-care assay. J Infect Dis. 2018;217(12):1889-96.

18. AASLD, EASL, APASL, ALEH. Call to action for liver associations to advance progress towards viral hepatitis elimination: a focus on simplified approaches to HCV testing and cure. Boston: AASLD; 2019.

19. Bourliere M, Gordon SC, Flamm SL, Cooper CL, Ramji A, Tong $\mathrm{M}$, et al. Sofosbuvir, velpatasvir, and voxilaprevir for previously treated HCV infection. N Engl J Med. 2017;376(22):2134-46.

20. World Health Organisation Regional Office for the Eastern Mediterranean (WHO EMRO). Viral hepatitis. Available at http://www. emro.who.int/irq/programmes/hepatitis.html. Accessed 29 Apr 2019.

21. Thein HH, Yi Q, Dore GJ, Krahn MD. Estimation of stagespecific fibrosis progression rates in chronic hepatitis $\mathrm{C}$ virus infection: a meta-analysis and meta-regression. Hepatology. 2008;48(2):418-31.
22. Polaris Observatory HCV Collaborators. Global prevalence and genotype distribution of hepatitis $C$ virus infection in 2015: a modelling study. Lancet Gastroenterol Hepatol. 2017;2(3):161-76.

23. United Nations. Department of Economic and Social Affairs, Population Division $(2007,2010,2018)$. Availabel at https://www. un.org/development/desa/pd/. Accessed 29 Apr 2019.

24. United Nations Statistics. Marriages and crude marriage rates (2007-2011). Available at https://unstats.un.org/unsd/demog raphic/products/dyb/dyb2011/Table23.pdf. Accessed 29 Apr 2019.

25. World Health Organisation (WHO). Iraq, World Blood Donor Day. 2012. Available at http://www.emro.who.int/irq/iraq-events/ world-blood-donor-day.html. Accessed 29 Apr 2019.

26. U.S. Department of Commerce. Healthcare resource guide: Iraq 2018. Available at https://2016.export.gov/industry/health/healt hcareresourceguide/eg_main_116238.asp. Accessed 29 Apr 2019.

27. Townsend R, McEwan P, Kim R, Yuan Y. Structural frameworks and key model parameters in cost-effectiveness analyses for current and future treatments of chronic hepatitis C. Value Health. 2011;14(8):1068-77.

28. McEwan P, Ward T, Yuan Y, Kim R, L'Italien G. The impact of timing and prioritization on the cost-effectiveness of birth cohort testing and treatment for hepatitis C virus in the United States. Hepatology. 2013;58(1):54-64.

29. Mangia A, Milligan S, Khalili M, Fagiuoli S, Shafran S, Carrat F, et al. GS-03-Global real world evidence of sofosbuvir/velpatasvir as a simple, effective regimen for the treatment of chronic hepatitis $\mathrm{C}$ patients: integrated analysis of 12 clinical practice cohorts. J Hepatol. 2019;70(1):e2-3.

30. XE Corporation. XE currency converter: live rates: 1 USD to IQD. 2019. https://www.xe.com/currencyconverter/convert/?Amount= $1 \&$ From $=$ USD \& To=IQD. Accessed 12 Jul 2021.

31. United States Department of Labor: Bureau of Labor Statistics. Databases, tables \& calculators by subject. 2019. https://data. bls.gov/timeseries/CUUR0000SAM?output_view=pct_12mths. Accessed 12 Jul 2021.

32. Eddy DM, Hollingworth W, Caro JJ, Tsevat J, McDonald KM, Wong JB. Model transparency and validation: a report of the ISPOR-SMDM modeling good research practices task force-7. Med Decis Mak. 2012;32(5):733-43.

33. Rein DB, Smith BD, Wittenborn JS, Lesesne SB, Wagner LD, Roblin DW, et al. The cost-effectiveness of birth-cohort screening for hepatitis $\mathrm{C}$ antibody in US primary care settings. Ann Intern Med. 2012;156(4):263-70.

34. Blach S, Schaetti C, Bruggmann P, Negro F, Razavi H. Costeffectiveness analysis of strategies to manage the disease burden of hepatitis C virus in Switzerland. Swiss Med Wkly. 2019;149:w20026.

35. Kim DY, Han K-H, Jun B, Kim TH, Park S, Ward T, et al. Estimating the cost-effectiveness of one-time screening and treatment for hepatitis C in Korea. PLoS One. 2017;12(1):e0167770.

36. Ruggeri M, Coretti S, Gasbarrini A, Cicchetti A. Economic assessment of an anti-HCV screening program in Italy. Value Health. 2013;16(6):965-72.

37. Selvapatt N, Ward T, Bailey H, Bennett H, Thorne C, See LM, et al. Is antenatal screening for hepatitis $\mathrm{C}$ virus cost-effective? A decade's experience at a London centre. J Hepatol. 2015;63(4):797-804.

38. Urbanus AT, van Keep M, Matser AA, Rozenbaum MH, Weegink CJ, van den Hoek A, et al. Is adding HCV screening to the antenatal national screening program in Amsterdam, the Netherlands, cost-effective? PLoS One. 2013;8(8):e70319.

39. World Health Organization. Progress report on access to hepatitis $\mathrm{C}$ treatment: focus on overcoming barriers in low- and middleincome countries. Geneva: World Health Organisation; 2018. 
40. Bennett H, McEwan P, Sugrue D, Kalsekar A, Yuan Y. Assessing the long-term impact of treating hepatitis $\mathrm{C}$ virus (HCV)-infected people who inject drugs in the UK and the relationship between treatment uptake and efficacy on future infections. PLoS One. 2015;10(5): 0125846

41. Bennett H, Gordon J, Jones B, Ward T, Webster S, Kalsekar A, et al. Hepatitis $\mathrm{C}$ disease transmission and treatment uptake: impact on the cost-effectiveness of new direct-acting antiviral therapies. Eur J Health Econ. 2017;18(8):1001-11.

42. World Health Organization. Hepatitis C. 2019. https://www.who. int/news-room/fact-sheets/detail/hepatitis-c. Accessed $12 \mathrm{Jul}$ 2021.
43. Boeke CE, Adesigbin C, Agwuocha C, Anartati A, Aung HT, Aung KS, et al. Initial success from a public health approach to hepatitis $\mathrm{C}$ testing, treatment and cure in seven countries: the road to elimination. BMJ Glob Health. 2020;5(12):e003767.

44. Martin NK, Vickerman P, Miners A, Foster GR, Hutchinson SJ, Goldberg DJ, et al. Cost-effectiveness of hepatitis C virus antiviral treatment for injection drug user populations. Hepatology. 2012;55(1):49-57. 